\title{
NOTE ON SOME OSCILLATION CRITERIA ${ }^{1}$
}

\author{
C. R. PUTNAM
}

1. The differential equation

$$
x^{\prime \prime}+f x=0,
$$

in which $f=f(t)$ is a continuous function on the half-line $0 \leqq t<\infty$, is said to be oscillatory if some (hence every) nontrivial solution $x=x(t)$ possesses an infinity of zeros on $0 \leqq t<\infty$ (clustering only at $+\infty$ ). Various criteria for the oscillatory nature of (1) are known; see, e.g., $[1]$ and the references cited there.

It was shown by Wintner [2] that if $F(t) \rightarrow \infty$ as $t \rightarrow \infty$, where

$$
F(t)=\int_{0}^{t} f(s) d s
$$

or even if

$$
G(t) \equiv t^{-1} \int_{0}^{t} F(s) d s \rightarrow \infty, \quad t \rightarrow \infty,
$$

then (1) must be oscillatory. Various refinements as well as variations of the criterion (3) were obtained by Hartman in [1]. The present note will be devoted to the derivation of two further criteria, given in $\left(^{*}\right)$ and $\left({ }^{* *}\right)$ below, involving the function $G(t)$ of (3).

Let $E(M, T)$ denote the set of points $t$ of the half-line $T \leqq t<\infty$ for which the function $G(t)$ of (3) satisfies the inequality $G(t)>M$, where $M$ is a positive constant. The following will be proved:

$\left(^{*}\right)$ Suppose that there exists a pair of sequences $T_{n}, M_{n}$ satisfying $T_{n} \rightarrow \infty, M_{n} \rightarrow \infty$ as $n \rightarrow \infty$, and for which

$$
\exp \left(M_{n} T_{n}\right) \text { meas } E\left(M_{n}, T_{n}\right) \rightarrow \infty, \quad n \rightarrow \infty .
$$

Then the equation (1) is oscillatory.

Since (3) implies meas $E(M, T)=\infty$ whenever $M, T>0$, the sufficiency of (3) for the oscillatory nature of (1) is a consequence of $(*)$. In fact, the proof of $\left(^{*}\right)$ will depend upon a refinement of the argument used by Wintner in [2] in obtaining the criterion (3).

It is known that if (3) is relaxed to

Received by the editors February 23, 1955.

1 This work was supported by the National Science Foundation research grant NSF-G481. 


$$
\lim \sup T^{-1} \int_{0}^{T} F(t) d t=\infty, \quad T \rightarrow \infty,
$$

then (1) need not be oscillatory; (see (II bis) of [1, p. 390]). It will be shown however, as a corollary of $\left({ }^{*}\right)$, that this situation cannot occur if, for instance, $f(t)$, or even $F(t)$, is bounded exponentially from below. Thus,

$\left.{ }^{* *}\right)$ If, in addition to (5), the function $F(t)$ of (2) also satisfies

$$
F(t)>-\exp (C t),
$$

for some positive constant $C$, then the equation (1) is oscillatory.

2. Proof of $\left(^{*}\right)$. If $x=x(t)$ and $y=y(t)$ denote two linearly independent solutions of (1), it is clear from [2] that the equation (1) is oscillatory if and only if

$$
\int_{0}^{\infty}\left(x^{2}+y^{2}\right)^{-1} d t=\infty .
$$

In order to prove $\left({ }^{*}\right)$, suppose, if possible, that (1) is nonoscillatory. It will be shown that this assumption implies (7), hence a contradiction, and the proof of $\left(^{*}\right)$ will be complete. Since, for large values of $t$, the logarithmic derivative, $z$, of a solution of (1) satisfies the Riccati equation $z^{\prime}+z^{2}+f=0$, the inequality $x^{2}+y^{2} \leqq$ const. $\exp \left(-2 \int_{0}^{t} F(s) d s+K t\right)$, in which $K$ denotes a constant, holds for $0 \leqq t<\infty ;$ cf. formula line (7) of [2]. Consequently,

$$
\int_{0}^{\infty}\left(x^{2}+y^{2}\right)^{-1} d t \geqq \text { const. } \int_{0}^{\infty} \exp [(2 G-K) t] d t,
$$

where $G=G(t)$ is defined by (3). Let $M>K$. In view of (8) and the inequalities

$$
\begin{aligned}
\int_{0}^{\infty} \exp [(2 G-K) t] d t & \geqq \int_{T}^{\infty} \exp [(2 G-K) t] d t \\
& \geqq \exp [(2 M-K) T] \text { meas } E(M, T),
\end{aligned}
$$

it follows that

$$
\int_{0}^{\infty}\left(x^{2}+y^{2}\right)^{-1} d t \geqq \text { const. } \exp (M T) \text { meas } E(M, T) .
$$

Since the left side of the inequality (9) is independent of $M$ and $T$, relation (4) implies (7). This completes the proof of $\left(^{*}\right)$. 
3. Proof of $\left({ }^{* *}\right)$. In view of $(6)$ and the relation $(t G)^{\prime}=F$, the inequality

$$
(t G)^{\prime}>-\exp (C t)
$$

holds for some positive constant $C$. If $a \leqq t \leqq b$ and $G(a)>0$, a quadrature of (10) leads to $t G(t)-a G(a)>-\int_{a}^{t} \exp (C s) d s>-(b-a)$ $\cdot \exp (C b)$, and hence

$$
G(t)>a b^{-1} G(a)-a^{-1}(b-a) \exp (C b), \quad a \leqq t \leqq b .
$$

According to (5), there exists a sequence $t=t_{1}<t_{2}<\cdots$ such that $t_{n} \rightarrow \infty$ and $G\left(t_{n}\right) \rightarrow \infty$ as $n \rightarrow \infty$. For a given $M>0$, choose $a=t_{n}$ (for some $n$ depending on $M$ ) such that $G\left(t_{n}\right)>2 M$, and let $b$ be defined by $b-a=\exp (-C b)$. Then relation (11) implies $G(t)>2 a b^{-1} M-a^{-1}$; hence, since $b-a \rightarrow 0$ as $a \rightarrow \infty, G(t)>M$ for $a \leqq t \leqq b$ and $a$ sufficiently large. Consequently, the inequality

$$
\exp (M a) \text { meas } E(M, a) \geqq \exp (M a-C b)
$$

holds for certain arbitrarily large numbers $a$ and $b=a+\exp (-C b)$. Clearly, for every fixed $M>C$, exp $(M T)$ meas $E(M, T) \rightarrow \infty$ for a sequence of $T(=a)$ values tending to $\infty$. In particular, relation (4) holds and $(*)$ now implies $(* *)$.

\section{REFERENCES}

1. P. Hartman, On nonoscillatory linear differential equations of second order, Amer. J. Math. vol. 74 (1952) pp. 389-400.

2. A. Wintner, A criterion of oscillatory stability, Quarterly of Applied Mathematics vol. 7 (1949) pp. 115-117.

Purdue University 